\title{
Thermoplasmonic Semitransparent Nanohole Electrodes
}

Daniel Tordera, Dan Zhao, Anton Volkov, Xavier Crispin and Magnus J onsson

The self-archived version of this journal article is available at Linköping University Electronic Press:

http:// urn.kb.se/ resolve?urn=urn:nbn:se:liu:diva-138266

N.B.: When citing this work, cite the original publication.

Tordera, D., Zhao, D., Volkov, A., Crispin, X., J onsson, M., (2017), Thermoplasmonic Semitransparent Nanohole Electrodes, Nano letters (Print), 17(5), 3145-3151.

https:// dx.doi.org/ 10.1021/ acs.nanolett.7b00574

Original publication available at:

https:// dx.doi.org/ 10.1021/acs.nanolett.7b00574

Copyright: American Chemical Society

http:// pubs.acs.org/ 


\section{Thermoplasmonic Semitransparent Nanohole Electrodes}

Daniel Tordera, Dan Zhao, Anton V. Volkov, Xavier Crispin, Magnus P. Jonsson*

Laboratory of Organic Electronics, Linköping University, SE-601 74 Norrköping, Sweden

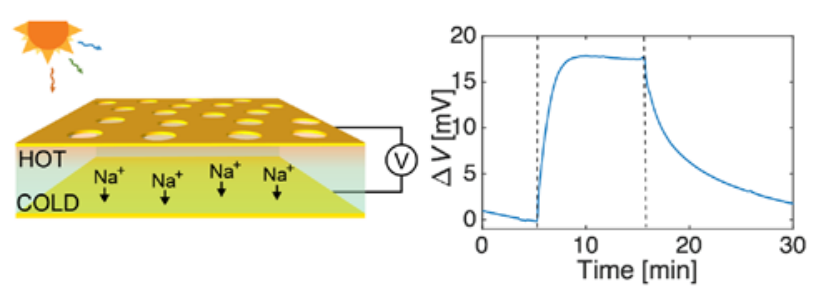




\section{ABSTRACT}

Non-radiative decay of plasmons in metallic nanostructures offers unique means for light-to-heat conversion at the nanoscale. Typical thermoplasmonic systems utilize discrete particles, while metal nanohole arrays were instead considered suitable as heat sinks to reduce heating effects. By contrast, we show for the first time that, under uniform broadband illumination (e.g. the sun), ultrathin plasmonic nanohole arrays can be highly competitive plasmonic heaters and provide significantly higher temperatures than analogous nanodisk arrays. Our plasmonic nanohole arrays also heat significantly more than non-structured metal films, while simultaneously providing superior light transmission. Besides being efficient light-driven heat sources, these thin perforated gold films can simultaneously be used as electrodes. We used this feature to develop "plasmonic thermistors" for electrical monitoring of plasmon-induced temperature changes. The nanohole arrays provided temperature changes up to $7.5 \mathrm{~K}$ by simulated sunlight, which is very high compared to previously reported plasmonic systems under similar conditions (solar illumination and ambient conditions). Both temperatures and heating profiles quantitatively agree with combined optical and thermal simulations. Finally, we demonstrate the use of a thermoplasmonic nanohole electrode to power the first hybrid plasmonic ionic thermoelectric device, resulting in strong solar-induced heat gradients and corresponding thermoelectric voltages.

KEYWORDS: thermoplasmonics, nanoholes, arrays, thermoelectric, electrodes, thermistor

Noble metal nanostructures interact strongly with light through excitation of plasmons (collective electron oscillations). ${ }^{1,2}$ This results in remarkable optical properties, including the possibility for 
light-to-heat conversion based on non-radiative plasmon decay. The corresponding research topic thermoplasmonics ${ }^{3,4}$ studies the use of plasmonic materials to control the temperature at the nanoscale, ${ }^{5-9}$ with applications found in a wide array of fields like cancer therapy, ${ }^{10-12}$ drug delivery, ${ }^{13-}$ ${ }^{15}$ nanochemistry, ${ }^{16-18}$ sensing, ${ }^{19-21}$ and energy, ${ }^{22,23}$ including thermoelectric applications. ${ }^{24-26}$ Most thermoplasmonic systems utilized different plasmonic nanoparticles as active elements. By contrast, continuous plasmonic systems like nanoholes in thin metal films ${ }^{27}$ were suggested suitable for applications were heating needs to be kept to a minimum. ${ }^{28}$ Indeed, a continuous (nanohole) film with high thermal conductivity can help to remove generated heat if a small area (micron-sized) is illuminated by focused light. However, we here show that that the behavior can be reversed for (non-focused) illumination of larger areas, for which plasmonic nanohole arrays instead can provide higher temperatures than typical surfaces based on plasmonic particles. These insights shed new light on using plasmonic nanohole arrays in thermoplasmonic applications based on the most abundant and cheap source of light, the sun. ${ }^{23}$ In addition, our findings may form important implications for temperature-sensitive applications like sensing, which is often based on uniform illumination of large $\left(\sim \mathrm{cm}^{2}\right)$ areas.

In this work, we demonstrate that large self-assembly based plasmonic nanohole arrays can be efficient light-driven sources of heat. Based on optical and heat transfer simulations, we show that nanohole arrays typically are superior absorbers than both nanodisk arrays with similar geometry (with respect to size and distribution of holes/disks) and flat films, while providing higher light transmission than the film. We study how the spacing between the nanostructures changes the optical properties of the plasmonic systems and find that the integrated solar absorption for nanoholes is higher than for nanodisks for all distances down to approximately $50 \%$ metal filling fraction.

Because the nanohole arrays are embedded in a continuous metal film, they can be simultaneously used as absorbers and electrodes. ${ }^{29,} 30$ This allowed patterning of the nanohole arrays into thermistor 
structures and electrical monitoring of temperature variations through changes in resistance of the metal film. Similar principles were previously employed to measure temperature-changes in nanowires connected to larger electrodes, ${ }^{31}$ nanohole films heated by a DC current, ${ }^{32}$ and plasmonic antennas positioned at the opening of a nanopore. ${ }^{33-35}$ Our experiments confirm that the nanohole arrays are highly competitive plasmonic heaters, with a temperature increase that is in good agreement with simulations. Finally, we incorporate a nanohole film as the hot electrode of an ionic thermoelectric cell, creating the first plasmon-driven ionic thermoelectric device. Simulated solar illumination created a significant difference of temperature between the hot (plasmonic) electrode and a colder bottom electrode, and a corresponding thermal voltage. This first demonstration of powering an ionic thermoelectric cell demonstrates that plasmonic nanoholes are highly suitable as combined heaters and electrodes, which may find use in a wide range of applications, not least in the field of solar energy harvesting.

We first theoretically evaluate the optical and heat transfer properties of gold nanohole arrays, nanodisk arrays, and a flat gold film. The nanoholes are $176 \mathrm{~nm}$ in diameter and distributed with a spacing (centre-to-centre) of $305 \mathrm{~nm}$ (choice based on image analysis of real samples, see below and in Supporting Information, $\mathrm{SI}$ ). As suitable comparative nanodisk systems, we use both the inverse structure (176 $\mathrm{nm}$ nanodisks), and also slightly smaller nanodisks $(130 \mathrm{~nm})$ for which the plasmonic absorption peak coincides with that of the nanoholes. These two nanodisk systems resemble traditional thermoplasmonic systems. ${ }^{3}$ All simulations employ the same overall geometry $(30 \mathrm{~nm}$ thick nanodisks/film over a glass substrate), and use the same simulation parameters (i.e. same boundary conditions, mesh size and source of light). 
a)

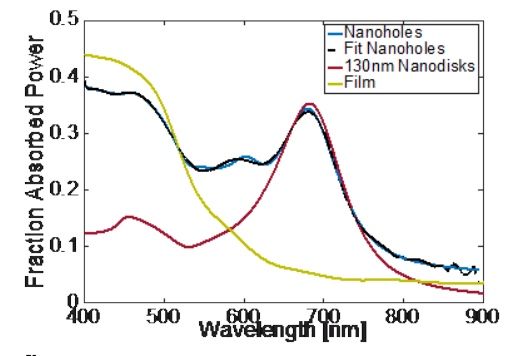

d)

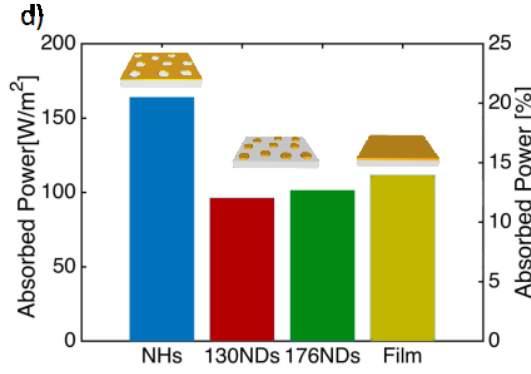

b)

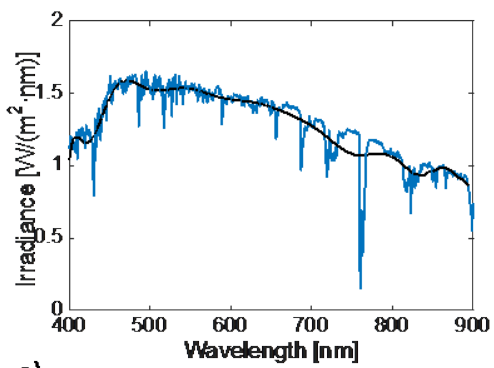

e) c)

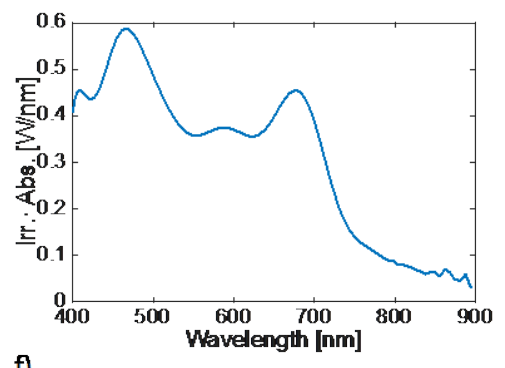

f)

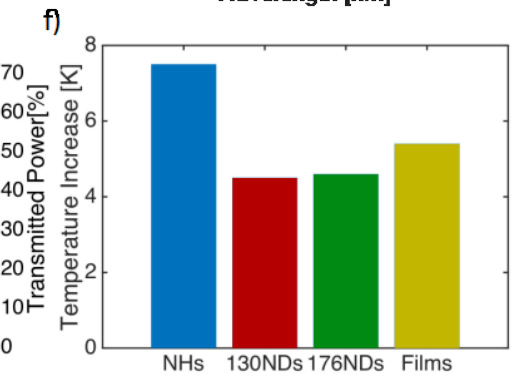

Figure 1. Optical and heat transfer simulations. (a) Fraction of absorbed power for the nanohole array (NHs, blue), $130 \mathrm{~nm}$ disks (NDs, red) and film (yellow), and polynomial fitting of the power absorbed for the nanohole array (black dashed line). (b) Spectrum of the sun with an intensity of 1 Sun (blue) and fitting of the spectrum (black dashed line). (c) Multiplication of the polynomial fits of the fraction of absorbed power of nanohole arrays and the spectrum of the sun. (d) and (e) Absorbed power and transmitted for the four studied systems. (f) Temperature increase for the four studied systems.

Extinction, absorption and reflection spectra for all four systems (nanohole array, the two types of nanodisks and the film) can be found on Figure S1 in the SI. The results show strong absorption for the nanohole array, and in a larger wavelength region than for the other systems. This is exemplified in Figure 1a for the two systems with coinciding absorption peaks (nanoholes in blue, nanodisks in red) and for the reference film (yellow). While absorbing about the same as the nanodisks in the region around the plasmonic absorption peaks, the nanoholes provide strong absorption also at short wavelengths, effectively combining the absorption of the nanodisks and the flat film. This 
means that plasmonic nanohole systems will be particularly suitable as absorbers for applications utilizing broadband light sources like the sun.

In order to evaluate total absorbed power upon solar illumination, we first multiply (polynomial fits of) the absorption spectra and the solar spectrum (1 Sun, $1000 \mathrm{~W} / \mathrm{m}^{2}$, see Figure $1 \mathrm{~b}$ ), as exemplified for the nanoholes in Figure 1c. Integrating this curve over the wavelength region of interest (400$1200 \mathrm{~nm}$ ) provides the total absorbed power per area. This gives $162 \mathrm{~W} / \mathrm{m}^{2}$ absorbed power by the nanohole array. With a total irradiance of the sun of $790 \mathrm{~W} / \mathrm{m}^{2}$ in this wavelength region, this corresponds to an absorption of $20.5 \%$ of the sunlight. We follow the same methodology to calculate the power that is absorbed, transmitted (including forward scattering) or reflected (including backscattering) for the nanohole array, the two nanodisk systems, and for the gold film (Figure 1d, 1e and Figure S2). We find that the nanohole array absorbs over $60 \%$ more power than both nanodisk systems ( $95 \mathrm{~W} / \mathrm{m}^{2}$ and $100 \mathrm{~W} / \mathrm{m}^{2}$ for the $130 \mathrm{~nm}$ and $176 \mathrm{~nm}$ nanodisks, respectively). This means that plasmonic nanohole films can be significantly stronger absorbers compared with the more traditional thermoplasmonic nanodisk structures. The nanoholes also absorb more power than the reference film $\left(110 \mathrm{~W} / \mathrm{m}^{2}\right)$, while at the same time providing superior transmittance (see Figure 1e). Indeed, the nanohole arrays are semi-transparent, transmitting $314 \mathrm{~W} / \mathrm{m}^{2}$ or around $40 \%$ of the sunlight in this region. We attribute the combined strong absorption and high transmittance to efficient coupling of light to plasmonic modes of the hole film, ${ }^{36}$ which reduces reflection in favor of both absorption and transmission (forward scattering) (see reflection spectra in Figure S1c and reflected power in Figure S2). As expected, the transmitted power is lower for the nanohole array compared with the $130 \mathrm{~nm}\left(562 \mathrm{~W} / \mathrm{m}^{2}\right)$ and the $176 \mathrm{~nm}\left(438 \mathrm{~W} / \mathrm{m}^{2}\right)$ nanodisk systems. However, the nanohole film still transmits over a factor two better than the flat film $\left(143 \mathrm{~W} / \mathrm{m}^{2}\right)$, which could be beneficial for applications where the combination of transparency and electrical conductivity is important. The total reflected power for the nanoholes (40\%) lies in between the power reflected for the nanodisk arrays (17\% and $32 \%$ for 130 and $176 \mathrm{~nm}$, respectively) and for the continuous film 
(68\%).

a)

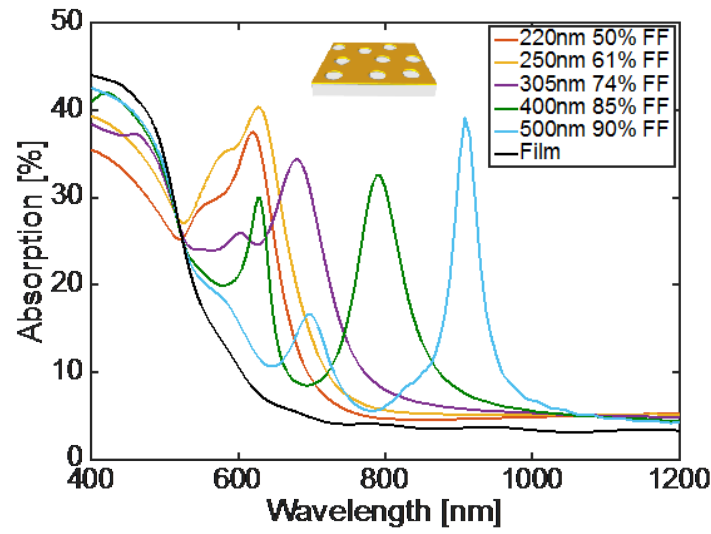

c)

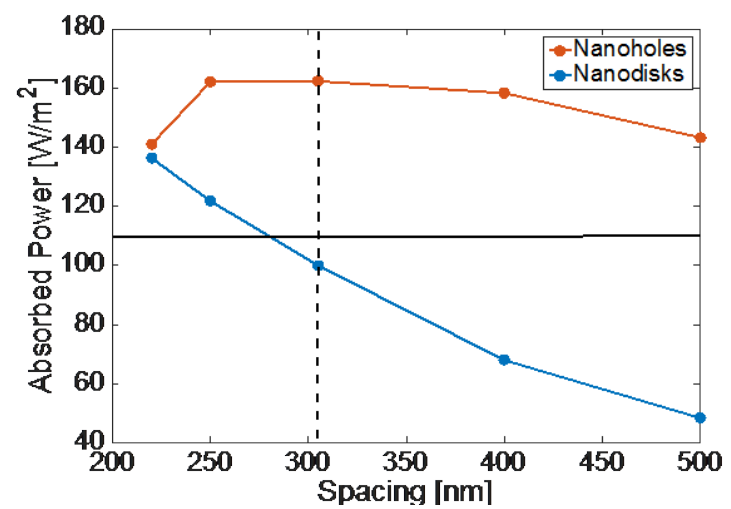

b)

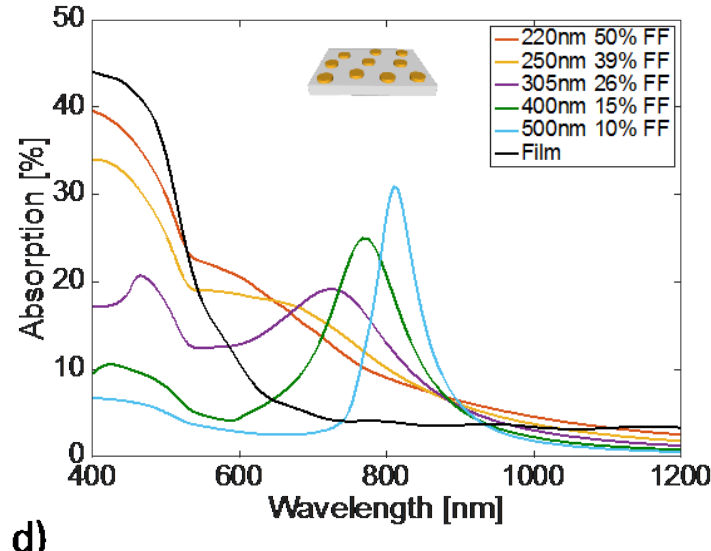

d)

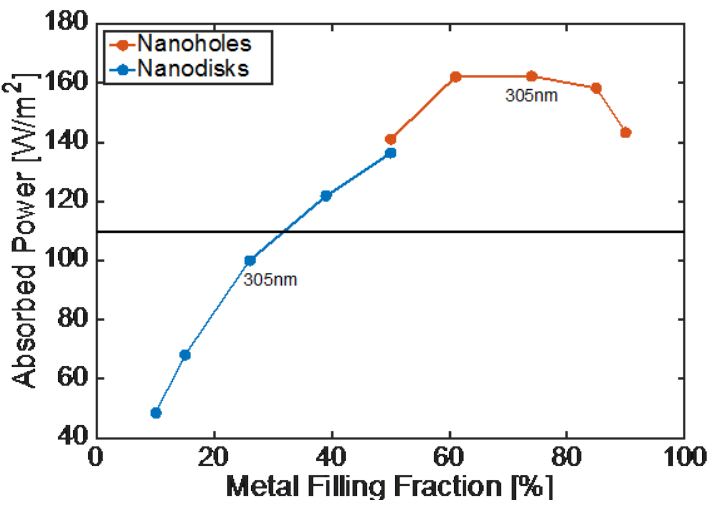

Figure 2. a) Absorption for gold nanohole arrays with different spacing. b) Absorption for gold nanodisk arrays with different spacing. Both holes and disks were $176 \mathrm{~nm}$ in diameter and $30 \mathrm{~nm}$ thick. FF corresponds to metal filling fraction. c) Absorbed power (solar-integrated) versus characteristic spacing for the nanohole arrays (red) and the nanodisk arrays (blue). The black line marks the absorbed power for the gold film. d) Absorbed power versus metal filling fraction (\%) for nanoholes (red) and nanodisks (blue). The black line marks the absorbed power for the gold film.

Next, we investigate how the spacing between nanostructures (centre-to-centre) influences the optical properties of the plasmonic arrays. Absorption and extinction spectra for arrays with different spacing are presented for the nanoholes in Figure 2a and S3a, respectively, and for the $176 \mathrm{~nm}$ nanodisks in Figure $2 \mathrm{~b}$ and S3b, respectively. As expected, the absorption peaks red-shift with 
increasing spacing for both nanoholes and nanodisks. ${ }^{37}$ This highlight the excellent spectral control and tunability of plasmonic systems, as opposed to other absorbers such as graphite powder. For example, the plasmonic absorber systems could be tuned to absorb (near) infrared light while transmitting light in the visible region, or to absorb in complementary regions to solar cells for improved functionality and/or efficiency.

Figure $2 \mathrm{c}$ show the integrated absorbed solar power for nanohole (red) and nanodisk (blue) arrays with different spacing, as well as for the flat gold film (black). Notably, the nanohole arrays provide highest absorption for all studied spacings, although the difference compared with the nanodisk array is very low for the shortest spacing of $220 \mathrm{~nm}$. Interestingly, this corresponds to a metal filling fraction (ratio between metal/non-metal) of $50 \%$ for both systems. This can also be seen from Figure $2 \mathrm{~d}$, which presents the relation between absorption and metal filling fraction. However, we stress that the absorption is not a mere result of the amount of metal on the surface, but an intricate balance between several factors and phenomena, as discussed in detail below.

All studied nanohole arrays absorbed better than the flat gold film, with values decreasing towards that of the gold film for very sparse arrays (see also for spacings up to $1000 \mathrm{~nm}$ in Figure S3c). Very dense nanohole arrays also showed decreased absorption. Spectral investigation shows that the decrease for dense arrays was most pronounced at low wavelengths (see Figure 2a), implying high influence from the lowered metal film coverage. Maximum integrated solar absorption for this nanohole geometry occurs at an optimum spacing of around $305 \mathrm{~nm}$. This optimum system provides high coverage of nanoholes to efficiently couple incident light to plasmons for subsequent absorption in the gold, while also maintaining a high metal coverage to promote absorption by the metal. Moreover, the optimum nanohole array provides a strong resonant plasmonic absorption in a region where the solar irradiance is particularly high. The nanodisk systems instead show a more monotonic decrease in absorption with increasing spacing. This is in agreement with the corresponding decrease in metal filling fraction and coverage of nanodisk absorbers on the surface. 
We also note that the spectral response (Figure $2 b$ ) indicates that the behavior of dense nanodisk arrays resembles the absorption of the continuous gold film, and also that relatively sparse nanodisk arrays (500 nm spacing) still absorb efficiently, but in a region of lower solar irradiance such that it contributes less to the integrated solar absorption.

To evaluate how the absorption of the optimum systems ( $305 \mathrm{~nm}$ spacing) translates to heating, we import the absorbed powers from the optical simulations as heat sources in finite element heat transfer simulations (Comsol Multiphysics, see Methods for details). For each system we use the same geometry as for the optical simulations (same nanohole/nanodisk sizes and a unit cell of $305 \mathrm{x}$ $305 \mathrm{~nm}$ ), but including the full thickness of the $1 \mathrm{~mm}$ thick glass substrate. We add the absorbed power as a uniformly distributed heat source in the gold parts of respective unit cell (in the metal forming the nanohole or in the nanodisk). The use of uniform heat sources is motivated by the significantly higher thermal conductivity of the gold compared with neighboring materials, making the heat rapidly distribute evenly throughout the gold (also for non-uniform heat sources) before spreading to the surroundings. ${ }^{28}$ This was also confirmed trough control simulations based on restricting the heat source to a smaller region of the gold, which showed no observable differences compared with using a uniform heat source. We employ periodic boundary conditions in the plane of the nanohole array to model uniform illumination of large (infinite) arrays. Heat flow from top and bottom of the systems was modeled by convective heat flux boundary conditions using a heat transfer coefficient of $10 \mathrm{~W} /\left(\mathrm{m}^{2} \cdot \mathrm{K}\right)$, as commonly used for ambient environments. ${ }^{38-40}$ Figure $1 \mathrm{f}$ presents the calculated heating for all four systems. The nanohole array temperature increases by $7.5 \mathrm{~K}$, compared to only $4.5,4.6$, and $5.4 \mathrm{~K}$ for the $130-\mathrm{nm}$ and $176-\mathrm{nm}$ diameter nanodisks and the reference film, respectively. This verifies that the higher absorption of the nanohole arrays also results in correspondingly higher heating than for the nanodisks. The results are in stark contrast to focused light conditions, for which similar nanoholes were shown to heat much 
less than nanodisk systems despite stronger absorption. ${ }^{28}$ Hence, illumination conditions are essential in determining the most suitable thermoplasmonic system for any given application. Our theoretical results are highly encouraging for adopting plasmonic nanohole arrays for light-to-heat conversion from solar illumination.

Next, we prepare plasmonic nanohole arrays and examine their function for high-efficiency thermoplasmonics. Self-assembly based colloidal lithography enabled fast and simple fabrication of gold films perforated with large arrays $\left(>1 \mathrm{~cm}^{2}\right)$ of short-range ordered nanoholes. ${ }^{41-43}$ Details of the experimental fabrication can be found in the Materials and Methods Section in the SI. In brief, 159 nm diameter polystyrene nanoparticles were deposited onto a glass substrate followed by evaporation of $30 \mathrm{~nm}$ gold and subsequent removal of the nanoparticles by tape stripping. This leaves a nanohole array pattern in the gold film. We also prepared flat reference films by evaporating $30 \mathrm{~nm}$ gold films on glass substrates.
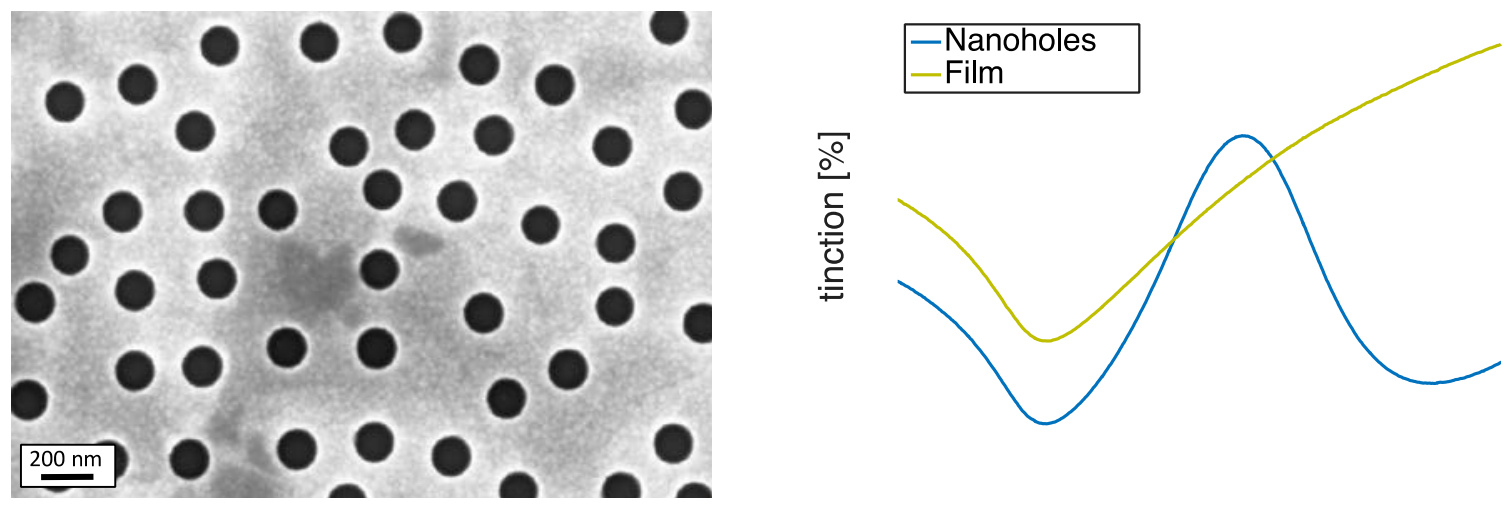

Figure 3. Characterization of plasmonic nanohole arrays. (a) SEM image of the nanohole array prepared by colloidal lithography. (b) Experimental extinction spectra of the nanohole array (blue) and the flat reference film (yellow).

Figure 3a shows a scanning electron microscope (SEM) image of a typical plasmonic nanohole array. From a set of SEM images, we determine an average hole-diameter of $176 \mathrm{~nm}$. We also use a radial 
distribution function (RDF) to calculate the distance between the holes, which gives a short-range order distribution with a characteristic spacing of $305 \mathrm{~nm}$ (Figure S4). This spacing corresponds to the optimum spacing for maximum integrated solar absorption, as determined from the simulations above. Figure $3 \mathrm{~b}$ presents the extinction spectra for the nanohole array (blue) and the reference gold film (yellow). The nanohole array shows an extinction maximum at $655 \mathrm{~nm}$ (SPP) and a minimum at $795 \mathrm{~nm}$ (localized hole resonance).$^{37}$ These values are very close to those predicted from the optical simulations, $658 \mathrm{~nm}$ for the SPP maximum and $745 \mathrm{~nm}$ for the localized resonance minimum (see Figure S1a). 
a)
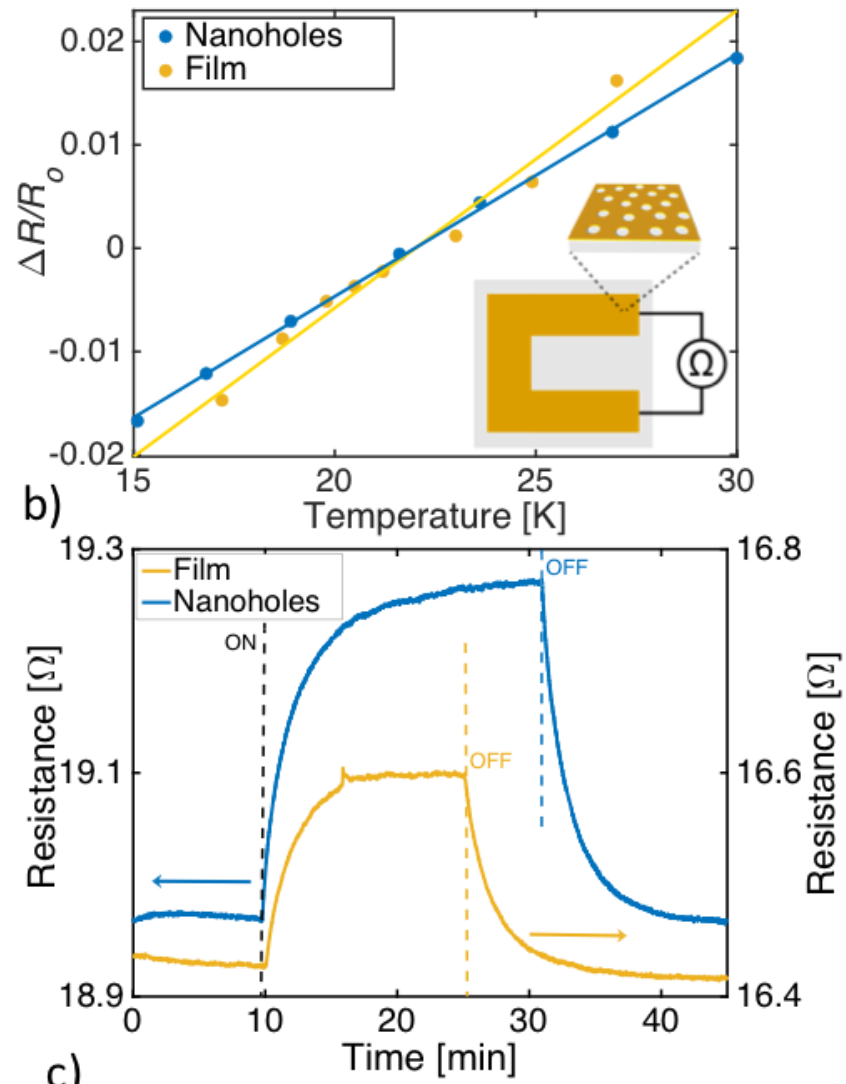

c)

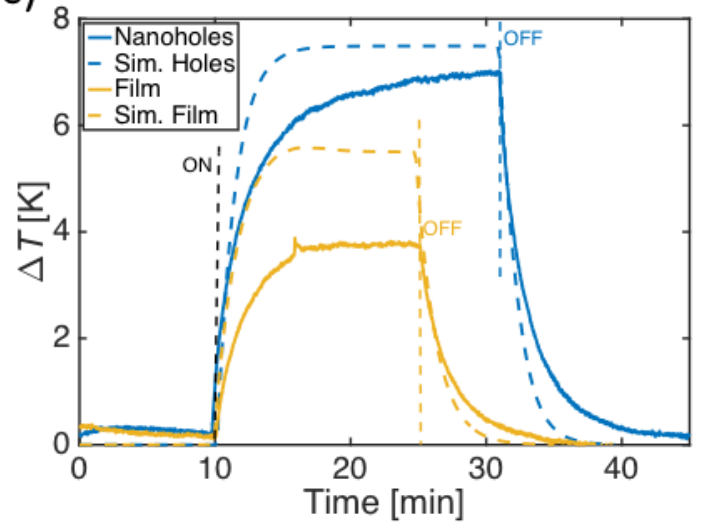

Figure 4. Plasmonic Thermistor Devices. (a) Calibration of nanohole array (blue dots) and film (yellow dots), with least-square lines for both systems. Inset: U-shape thermistor device schematic. (b) Resistance versus time under illumination for the nanohole array (blue, left axis) and film (yellow, right axis). Horizontal dashed lines indicate switch on and off of the light. (c) Temperature increase versus time under illumination for the nanohole array (experimental: blue, simulated: blue dashed line) and film (experimental: yellow, simulated: yellow dashed line). Horizontal dashed lines indicate switch on and off of the light. 
Patterning the nanohole arrays into $U$ shapes allowed us to connect electrodes at each end of the film and use them as "plasmonic thermistors". These devices enable electrical monitoring of temperature changes in the plasmonic structure, based on the temperature-dependence of the gold resistivity. The principle utilizes a fundamental advantage of nanohole arrays compared with discrete nanoparticle arrays in the sense that they form a continuous film and can be used as electrodes in addition to providing plasmonic functionalities. The thermistors were calibrated by varying the temperature of the plasmonic films using Peltier elements and measuring the corresponding changes in resistance using a sourcemeter. Figure 4a shows calibration curves of both a nanohole array and a reference thermistor without nanoholes. Possible variations in measured device resistance between measurements (e.g. due to small differences in the distance between contacting electrodes) were corrected for by plotting the normalized change in resistance, $\Delta R / R_{0}$, where $\Delta R$ is the change in resistance and $R_{0}$ is the resistance measured at room temperature $\left(22^{\circ} \mathrm{C}\right)$.

Light-induced heating of the nanohole arrays could now be investigated experimentally using the plasmonic thermistor devices. Figure $4 \mathrm{~b}$ shows the resistance versus time upon illumination with $\mathrm{a}$ solar simulator (at an irradiance of 1 Sun, $1000 \mathrm{~W} / \mathrm{m}^{2}$ ) for both the nanoholes (blue, right axis) and the film reference (yellow, left axis). For both devices, the resistance increases monotonically to a saturated value upon illumination, and decreases to the original resistance after the light is turned off. Figure $4 \mathrm{c}$ shows the corresponding temperature changes over time, as converted using the calibration curves. We can see that the nanohole arrays (blue) heat up almost double that of the reference film (yellow). The temperature increases are around 7.0 and $3.8 \mathrm{~K}$ for the nanohole arrays and the reference films, respectively. These values are in good agreement with the predicted values from the heat transfer simulations, 7.5 and $5.4 \mathrm{~K}$, respectively. Direct comparison of temperature increase for different plasmonic systems needs to be done with care, since it depends on the complete device geometry and external factors like the surrounding medium and light conditions. 
We therefore compare our results with a report of gold nanodisk arrays that use a similar device geometry (plasmonic arrays on glass substrate) and that also employ simulated solar illumination under ambient conditions. ${ }^{23}$ That work on $110-\mathrm{nm}$ in diameter and $20 \mathrm{~nm}$ thick nanodisk arrays showed heating of less than $3 \mathrm{~K}$ upon solar illumination (linearly extrapolated from reported values for illumination at 2 Sun). This corroborates our theoretical calculations and highlights that nanohole films are highly competitive alternatives as light-induced plasmonic heaters. Figure $4 c$ also includes results from time-dependent heat transfer simulations (blue and yellow dashed lines for the nanohole array and the gold film, respectively), which shows good consistency with the experimental results. We observe a higher maximum temperature for the simulations and slightly faster kinetics, which may be related to small variations in the exact properties of the material and thicknesses and deviations in the thermal flows from the experimental and theoretical systems. 
a)

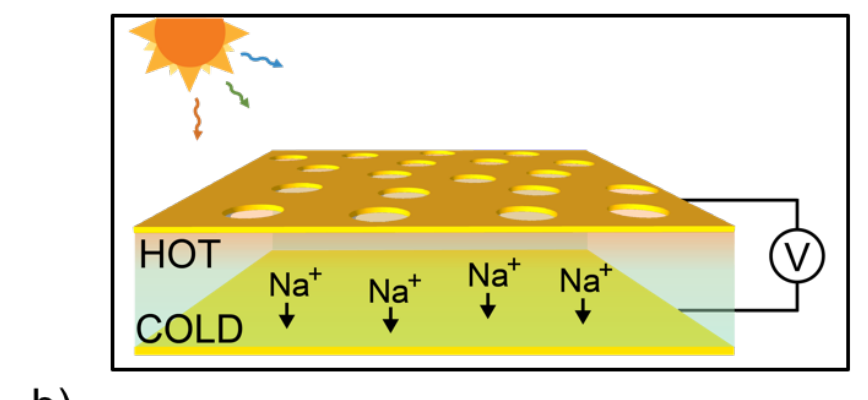

b)
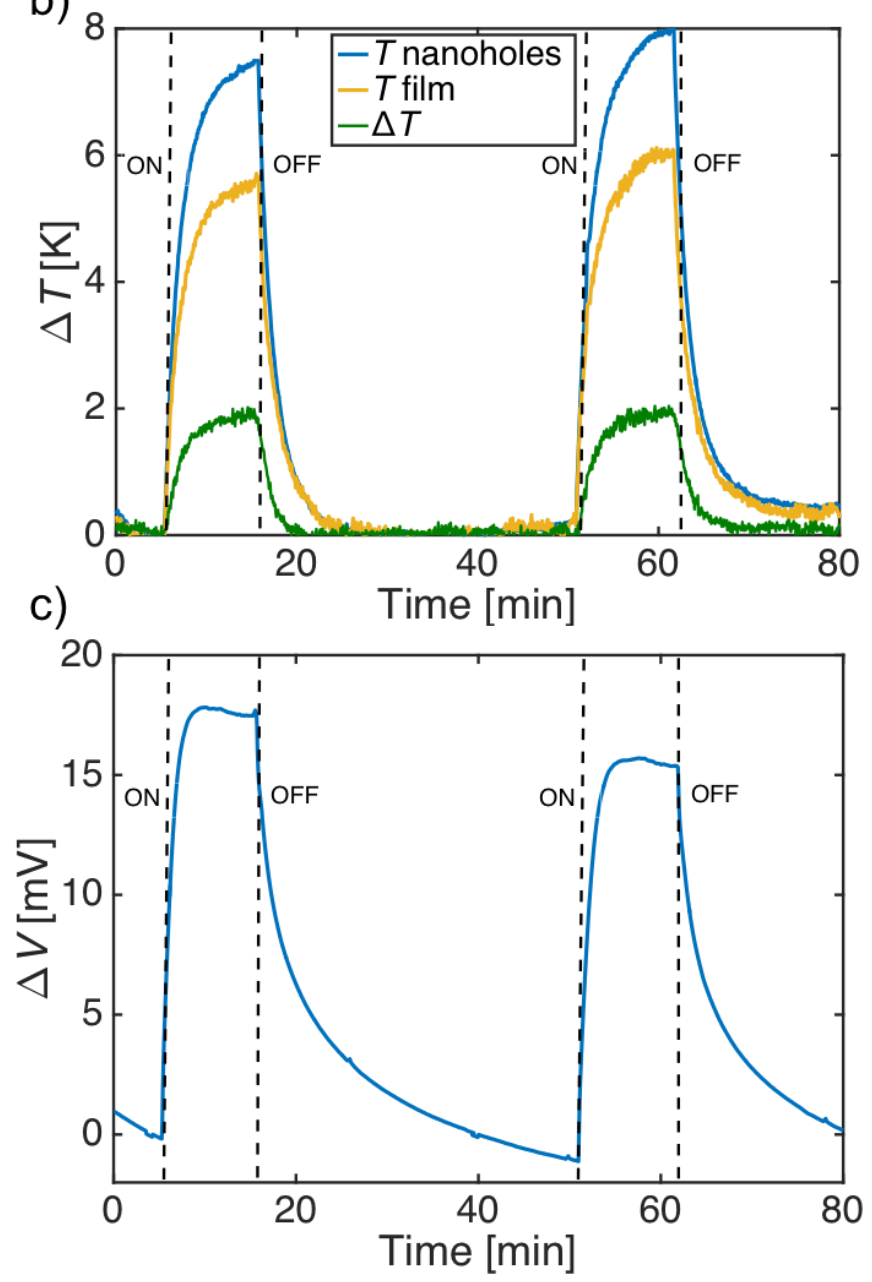

Figure 5. Hybrid plasmonic-ionic thermoelectric device. (a) Schematic view of the plasmonic ionic thermoelectric device (omitting the top and bottom glass substrates). (b) Measured temperature increase under illumination for the nanohole array top electrode (blue) and the gold film bottom electrode (yellow) in the ionic thermoelectric device, as well as the difference in temperature (green) between the two electrodes. The horizontal dashed lines indicate on and off switch of the 
illumination. (c) Measured thermovoltage for the ionic thermoelectric device. The horizontal dashed lines indicate switching on and off the illumination.

Finally, we demonstrate also in practical devices that nanohole arrays are highly suitable as combined plasmonic heaters and electrodes and report the first hybrid plasmon-driven ionic thermoelectric device. The concept uses solar-powered heating of a plasmonic nanohole array to create a temperature difference $(\Delta T)$ over an ionic thermoelectric film (see Figure $5 \mathrm{a}$ ). ${ }^{44}$ Through the thermoelectric effect, $\Delta T$ translates to a thermal voltage proportional to the Seebeck coefficient of the material. For this purpose, we used a $1 \mathrm{~mm}$ thick ionic thermoelectric layer based on sodium hydroxide-treated polyethylene glycol (NaOH-PEG), which has a very high Seebeck coefficient with reported values between $7 \mathrm{mV} / \mathrm{K}$ and $11 \mathrm{mV} / \mathrm{K} \cdot{ }^{44,45}$ Both the plasmonic top electrode and the gold film bottom electrode were patterned as thermistors to monitor temperature-changes on each electrode independently, while also allowing measurements of the Seebeck voltage over the film. We illuminated the device from the backside of glass slide with the nanohole array. Besides ensuring maximum light exposure of the nanohole array, we also found that backward illumination results in higher absorption compared with front illumination (see simulations in Figure S5), which may be related to lower reflection at the glass-gold interface compared to the air-gold interface. Figure $5 \mathrm{~b}$ shows the measured temperature increase for both the nanohole array electrode (blue) and the gold film bottom electrode (yellow) when illuminated with simulated sunlight. The green curve presents the temperature difference between the electrodes. As expected, the temperature increase is higher for the nanoholes (ca. $8 \mathrm{~K}$ ) than for the bottom electrode $(c a .6 \mathrm{~K})$, generating a $\Delta T$ between the two electrodes of around $2 \mathrm{~K}$. The heating of the bottom electrode is likely a combination of heat flow from the top electrode and direct heating by light transmitted through the nanohole film and the ionic thermoelectric layer.

Figure $5 \mathrm{c}$ presents the thermovoltage induced by the temperature difference over the thermoelectric film. The thermovoltage saturates at $17.8 \mathrm{mV}$, which is in agreement with $18 \mathrm{mV}$ as predicted based 
on $2 \mathrm{~K}$ heating and the average reported Seebeck coefficient of $9 \mathrm{mV} / \mathrm{K}$ for the ionic thermoelectric material. ${ }^{44,45}$ The kinetics of the thermovoltage mainly follows the evolution of $\Delta T$, albeit with a slower decay. This may be due to slow equilibration and movement of the ionic charge carriers in the electrolyte film. The results for this hybrid device also indicates a slightly faster change in thermovoltage than $\Delta T$, which was not observed upon conventional heating for similar ionic thermoelectric devices. ${ }^{44,45}$ Such effect would be highly interesting and could possibly be related to the plasmonic heating process or the asymmetric structure of the electrodes. Future systematic investigations focusing on the detailed kinetics may provide better understanding of the thermovoltage evolution and possible additional contributions to the measured voltage. This demonstration of the first plasmon-driven ionic thermoelectric device highlights the strengths of plasmonic nanohole arrays as combined electrodes (here functioning as thermistors) and efficient light-to-heat converters, which could find important use in a variety of applications. The ultrathin plasmonic heaters are also compatible with large-scale fabrication and show additional advantages, including spectral control of the optical properties.

In summary, we have shown for the first time that plasmonic nanohole arrays can be highly efficient light-driven heat sources, with a temperature increase upon solar illumination that is much higher than for both comparative plasmonic nanodisk systems and non-structured metal films. Moreover, the nanohole arrays could be simultaneously used as plasmonic absorbers and electrodes, which enabled the development of plasmonic nanohole thermistors and electrical quantification of heating upon illumination with simulated sun light. The resulting measured temperature increase is in good agreement with predictions based on combined optical and heat transfer simulations. Finally, we used the nanohole arrays as hot plasmonic electrodes to create the first light-driven plasmonic ionic thermoelectric device. Simulated solar illumination induced a temperature difference of $2 \mathrm{~K}$ between the hot and cold electrodes, effectively generating a thermovoltage of $17.8 \mathrm{mV}$ that matches the prediction based on the Seebeck coefficient of the ionic thermoelectric material. These first important first steps towards thermoelectric light-harvesting using plasmonic nanohole arrays 
illustrate the unique possibility of using plasmonic nanohole arrays, with spectrally controlled absorption peaks, as combined electrodes and efficient thermoplasmonic thin films.

\section{ASSOCIATED CONTENT}

\section{Supporting Information}

The Supporting Information is available free of charge on the ACS Publication website at DOI: $X X X X X X X X X X X X$

Materials and methods (optical and heat transfer simulations, fabrication of nanohole array thermistors, fabrication of plasmonic ionic thermoelectric devices, optical and morphological characterization, thermistor characterization and optical measurements, thermoelectric device characterization). Simulated extinction, absorption and reflection of the different studied systems. Integrated reflected power. Extinction of nanodisks and nanoholes and total absorbed power for nanoholes at different spacing. Radial distribution function of the nanohole arrays. Absorption directionality of the nanohole arrays.

\section{AUTHOR INFORMATION}

\section{Corresponding Author}

*E-mail: magnus.jonsson@liu.se

\section{Notes}

The authors declare no competing interest. 
We would like to acknowledge Zia Ullah Khan for fruitful discussions and advice in the lab. We also thankfully acknowledge financial support from the Wenner-Gren Foundations, the Swedish Research Council, the Swedish Foundation for Strategic Research, the ÅForsk Foundation, Knut and Alice Wallenberg Foundation (Tail of the sun), and the Swedish Government Strategic Research Area in Materials Science on Functional Materials at Linköping University (Faculty Grant SFO-Mat-LiU No 2009 00971).

\section{REFERENCES}

1. Bohm, D.; Pines, D. Phys. Rev. 1953, 92, 609-625.

2. Murray, W. A.; Barnes, W. L. Adv. Mater. 2007, 19, 3771-3782.

3. Baffou, G.; Quidant, R. Laser Photon. Rev 2013, 7, 171-187.

4. De Sio, L.; Placido, T.; Comparelli, R.; Curri, M. L.; Striccoli, M.; Tabiryan, N.; Bunning, T. J. Prog. Quant. Electron. 2015, 41, 23-70.

5. Baffou, G.; Urena, E. B.; Berto, P.; Monneret, S.; Quidant, R.; Rigneault, H. Nanoscale 2014, 6, 8984-8989.

6. Coppens, Z. J.; Li, W.; Walker, D. G.; Valentine, J. G. Nano Lett. 2013, 13, 1023-1028.

7. Baffou, G.; Berto, P.; Bermúdez Ureña, E.; Quidant, R.; Monneret, S.; Polleux, J.; Rigneault, H. Acs Nano 2013, 7, 6478-6488.

8. Baffou, G.; Quidant, R.; Girard, C. Phys. Rev. B 2010, 82, 11.

9. Baffou, G.; Quidant, R.; de Abajo, F. J. G. Acs Nano 2010, 4, 709-716.

10. Cherukuri, P.; Glazer, E. S.; Curley, S. A. Adv. Drug Deliv. Rev. 2010, 62, 339-345.

11. Gobin, A. M.; Lee, M. H.; Halas, N. J.; James, W. D.; Drezek, R. A.; West, J. L. Nano Lett. 2007, 7, 1929-1934.

12. Hirsch, L. R.; Stafford, R. J.; Bankson, J. A.; Sershen, S. R.; Rivera, B.; Price, R. E.; Hazle, J. D.; Halas, N. J.; West, J. L. Proc. Natl. Acad. Sci. USA 2003, 100, 13549-13554.

13. Lin, L.; Peng, X.; Wang, M.; Scarabelli, L.; Mao, Z.; Liz-Marzán, L. M.; Becker, M. F.; Zheng, Y. Acs Nano 2016, 10, 9659-9668.

14. Han, G.; Ghosh, P.; De, M.; Rotello, V. M. NanoBiotechnology 2007, 3, 40-45.

15. Kang, H.; Kim, B.-G.; Na, H. B.; Hwang, S. J. Phys. Chem. C 2015, 119, 25974-25982.

16. Robert, H. M. L.; Kundrat, F.; Bermúdez-Ureña, E.; Rigneault, H.; Monneret, S.; Quidant, R.; Polleux, J.; Baffou, G. ACS Omega 2016, 1, 2-8. 
17. Baffou, G.; Quidant, R. Chem. Soc. Rev. 2014, 43, 3898-3907.

18. Baffou, G.; Polleux, J.; Rigneault, H.; Monneret, S. J. Phys. Chem. C 2014, 118, 4890-4898.

19. Baral, S.; Green, A. J.; Richardson, H. H. Acs Nano 2016, 10, 6080-6089.

20. Karker, N.; Dharmalingam, G.; Carpenter, M. A. Acs Nano 2014, 8, 10953-10962.

21. Green, A. J.; Alaulamie, A. A.; Baral, S.; Richardson, H. H. Nano Lett. 2013, 13, 4142-4147.

22. Mikhail, O.; Younes, R. d.; Constantin, S. J. Opt. 2015, 17, 125901.

23. Jonsson, G. E.; Miljkovic, V.; Dmitriev, A. Sci. Rep. 2014, 4, 5111.

24. Xiong, Y. J.; Long, R.; Liu, D.; Zhong, X. L.; Wang, C. M.; Li, Z. Y.; Xie, Y. Nanoscale 2012, 4, 4416-4420.

25. Komatsu, R.; Balčytis, A.; Seniutinas, G.; Yamamura, T.; Nishijima, Y.; Juodkazis, S. Sol Energ Mat Sol C 2015, 143, 72-77.

26. Pan, Y.; Tagliabue, G.; Eghlidi, H.; Höller, C.; Dröscher, S.; Hong, G.; Poulikakos, D. Sci. Rep. 2016, 6, 37564.

27. Jonsson, M. P.; Dahlin, A. B.; Jonsson, P.; Hook, F. Biointerphases 2008, 3, FD30-FD40.

28. Yang, Y.-J.; Lee, Y.-G. J. Appl. Phys. 2016, 119, 083108.

29. Jonsson, M. P.; Jonsson, P.; Hook, F. Anal. Chem. 2008, 80, 7988-7995.

30. Dahlin, A. B.; Jonsson, P.; Jonsson, M. P.; Schmid, E.; Zhou, Y.; Hook, F. Acs Nano 2008, 2, 2174-2182.

31. Herzog, J. B.; Knight, M. W.; Natelson, D. Nano Lett. 2014, 14, 499-503.

32. Virk, M.; Xiong, K. L.; Svedendahl, M.; Kall, M.; Dahlin, A. B. Nano Lett. 2014, 14, 3544-3549.

33. Jonsson, M. P.; Dekker, C. Nano Lett. 2013, 13, 1029-1033.

34. Nicoli, F.; Verschueren, D.; Klein, M.; Dekker, C.; Jonsson, M. P. Nano Lett. 2014, 14, 69176925.

35. Reiner, J. E.; Robertson, J. W. F.; Burden, D. L.; Burden, L. K.; Balijepalli, A.; Kasianowicz, J. J. J. Am. Chem. Soc. 2013, 135, 3087-3094.

36. Ebbesen, T. W.; Lezec, H. J.; Ghaemi, H. F.; Thio, T.; Wolff, P. A. Nature 1998, 391, 667-669.

37. Sannomiya, T.; Scholder, O.; Jefimovs, K.; Hafner, C.; Dahlin, A. B. Small 2011, 7, 1653-1663.

38. Stevens, J. W. The 34th Intersociety Energy Conversion Engineering Conference 1999, 012564, 2564.

39. Engineer's Edge, Convective Heat Transfer Coefficient Table Chart, http://www.engineersedge.com/heat transfer/convective heat transfer coefficients 13378.htm. (31st January 2017),

40. Engineering Toolbox, Overall Heat Transfer Coefficient, http://www.engineeringtoolbox.com/overall-heat-transfer-coefficient-d 434.html. (31st January 2017), 
41. Fredriksson, H.; Alaverdyan, Y.; Dmitriev, A.; Langhammer, C.; Sutherland, D. S.; Zäch, M.; Kasemo, B. Adv. Mater. 2007, 19, 4297-4302.

42. Hanarp, P.; Käll, M.; Sutherland, D. S. J. Phys. Chem. B 2003, 107, 5768-5772.

43. Dahlin, A. B.; Sannomiya, T.; Zahn, R.; Sotiriou, G. A.; Vörös, J. Nano Lett. 2011, 11, 13371343.

44. Zhao, D.; Wang, H.; Khan, Z. U.; Chen, J. C.; Gabrielsson, R.; Jonsson, M. P.; Berggren, M.; Crispin, X. Energ. Environ. Sci. 2016, 9, 1450-1457.

45. Zhao, D.; Fabiano, S.; Berggren, M.; Crispin, X. Nat. Commun. 2017, 8, 14214. 


\title{
Supporting Information to Thermoplasmonic Semitransparent Nanohole Electrodes
}

\author{
Daniel Tordera, Dan Zhao, Anton V. Volkov, Xavier Crispin, Magnus P. Jonsson* \\ Laboratory of Organic Electronics, Linköping University, SE-601 74 Norrköping, Sweden
}

\section{Materials and methods}

\section{Optical Simulations}

Optical simulations were done using the Finite-Difference Time-Domain (FDTD) methodology. For that purpose, we used the FDTD Solutions software package from Lumerical (version 8.15.736). The simulation size was $305 \mathrm{~nm}$ on $x$ and $y$, and $250 \mathrm{~nm}$ on $z$. Periodic boundary conditions were established on the $x$ and $y$ directions, and perfectly matched layers on the $z$ direction. The simulation comprised a glass substrate (refractive index 1.5, in the $x-y$ plane) and either a 30-nm thick gold film, a 30-nm thick gold film with a 176-nm diameter hole, a 176-nm diameter and 30-nm thick gold disk, or a $130-\mathrm{nm}$ diameter and $30-\mathrm{nm}$ thick gold disk on top. Gold permittivity was provided by the default material in the Lumerical software (Johnson \& Christie, 6 coefficients). The meshing over the gold film/disk was set to $2 \mathrm{~nm}$ on all the three axes. A plane-wave was used as a source, normal to the surface of the film/disks and with a wavelength range of 400 to $1200 \mathrm{~nm}$. Transmission and reflection monitors were used to extract the optical response of the different systems.

\section{Heat Transfer Simulations}

Heat transfer simulations were done using the Finite Element Method (FEM). For that purpose, we used the COMSOL-Multiphysics software package (version 5.1). The simulation size was $305 \mathrm{~nm}$ on $x$ and $y$, as in the optical simulations. We simulated the same film/nanodisk structures as detailed above for the optical simulations, with a glass substrate of $1 \mathrm{~mm}$, as used in the experiments. Periodic boundary conditions were used on $x$ and $y$ and a convective heat flux was used on $z$ (top and bottom of the simulation) with a heat transfer coefficient of $10 \mathrm{~W} /\left(\mathrm{m}^{2} \mathrm{~K}\right)$, a common value used for air. The material properties such as thermal conductivity, heat capacity and density of gold and glass were provided by the COMSOL-Multiphysics software package. Both stationary and time-dependent studies were carried out. For the time-dependent simulations explicit events were created at which the heat source was turned on or off (i.e. light on/off) in order to accurately monitor the change of temperature on the state change of the system. 


\section{Fabrication of Nanohole Array Thermistors}

The nanohole arrays were fabricated following the process of colloidal lithography. First, $3 \times 3 \mathrm{~cm}$ cover glasses with a thickness of $1 \mathrm{~mm}$ were cleaned by hand-rubbing with Mucasol soap (Z637181, Sigma-Aldrich), sonicating in Mucasol soap ( $5 \mathrm{~min}$ ), deionized (DI) $\mathrm{H}_{2} \mathrm{O}$ (5 min) and isopropanol (5 min). The substrates were dried with an $\mathrm{N}_{2}$ gun and treated with UV-Ozone (15 min). Colloidal lithography was done by first coating the surface with Poly(diallyldimethylammonium chloride) (522376, Sigma-Aldrich) diluted to $2 \mathrm{wt} . \%$ in $\mathrm{H}_{2} \mathrm{O}$ for $1 \mathrm{~min}$, followed by rinsing with $\mathrm{DI}^{\mathrm{H}_{2} \mathrm{O} \text { and }}$ drying with $\mathrm{N}_{2}$. Negatively charged polystyrene nanoparticles (158 nm, 0.1 wt.\% in $\mathrm{H}_{2} \mathrm{O}$, Microparticles $\mathrm{GmbH}$ ) were then deposited on the surface. The nanoparticles were left for $1 \mathrm{~min}$, resulting in a short-range order monolayer. The excess of nanoparticles was rinsed away with $\mathrm{H}_{2} \mathrm{O}$ and the sample was dried with $\mathrm{N}_{2}$. A mask was placed on the surface to create the thermistor pattern. $2 \mathrm{~nm}$ titanium $(0.1 \mathrm{~nm} / \mathrm{s}$ rate) were evaporated to increase adhesion followed by $30 \mathrm{~nm}$ gold $(0.1 \mathrm{~nm} / \mathrm{s}$ rate). The mask was removed manually and the polystyrene nanoparticles were removed by tape-stripping, resulting in the nanohole array structure. As reference, gold films were also prepared by following the same cleaning procedure and evaporation steps.

\section{Fabrication of Plasmonic lonic Thermoelectric Devices}

Polydimethylsiloxane (PDMS) of 1-mm thickness was prepared and cut into proper size. A thin layer of uncured PDMS was used as glue. Both the prepared glass substrates with gold film and nanohole arrays were glued to the PDMS spacer, and then baked at $70^{\circ} \mathrm{C}$ in an oven for 30 mins. A cavity of 84 $\mathrm{mm}^{3}$ was obtained. A $3 \mathrm{wt}$. \% solution based on sodium hydroxide and polyethylene glycol $(\mathrm{NaOH}-$ $\mathrm{PEG}$ ) was prepared and stirred before use. The solution was injected into the chamber.

\section{Optical and Morphological Characterization}

The extinction spectra of the gold nanohole arrays and the gold films were taken by using a UV/VIS/NIR Spectrometer (Lamba900, Perkin Elmer Instruments). Scanning Electron Microscope (SEM) images of the nanohole arrays were taken at different magnifications (10x, 20x, 50x, 100x, 250x). The size of the nanoholes was calculated by using a custom-made matlab script. The Radial Distribution Function (RDF) was calculated using the RDF macro of ImageJ.

\section{Thermistor Characterization and Optical Measurements}

For calibration, the samples were placed on a Peltier element and a thermocouple was used to measure the temperature of the surface of the sample. The temperature was changed and the resistance of the devices were measured and recorded in one-second intervals with a Keithley 2420 sourcemeter. The plasmonic thermistor response upon illumination was measured by using a LCS100 Solar Simulator from Oriel Instruments as the light source. The system uses a $100 \mathrm{~W}$ Xe lamp and an AM1.5G filter. The irradiance was set to 1 Sun $\left(1000 \mathrm{~W} / \mathrm{m}^{2}\right)$ using a calibrated reference cell and meter (91150-KG5 from Oriel Instruments). The resistance was measured and recorded over time 
with a Keithley 2420 sourcemeter. A custom-designed LabVIEW program was used to control the equipment and gather the data on a personal computer.

\section{Thermoelectric Device Characterization}

lasmonic ionic thermoelectric device response upon illumination was characterized using the aforementioned light source. In this case, the resistance of both the nanohole arrays (hot electrode) and the gold film (cold electrode) were measured and recorded over time using the front and rear channels of a Keithley 2420 sourcemeter. Simultaneously, the generated thermovoltage was measured and recorded using a Keithley 2182A nanovoltmeter. A custom-designed LabVIEW program was used to control the equipment and gather the data on a personal computer. 


\section{Simulated extinction, absorption and reflection}

We carried out optical simulations using the finite-difference time-domain (FDTD) method in order to calculate the extinction, absorption and reflection spectra of the four studied systems: 176-nm diameter 30-nm thick gold nanohole arrays (blue), 176-nm 30-nm thick gold nanodisks (green), 130$\mathrm{nm}$ 30-nm thick gold nanodisks (red) and 30-nm thick gold films (yellow) (Figure S1).
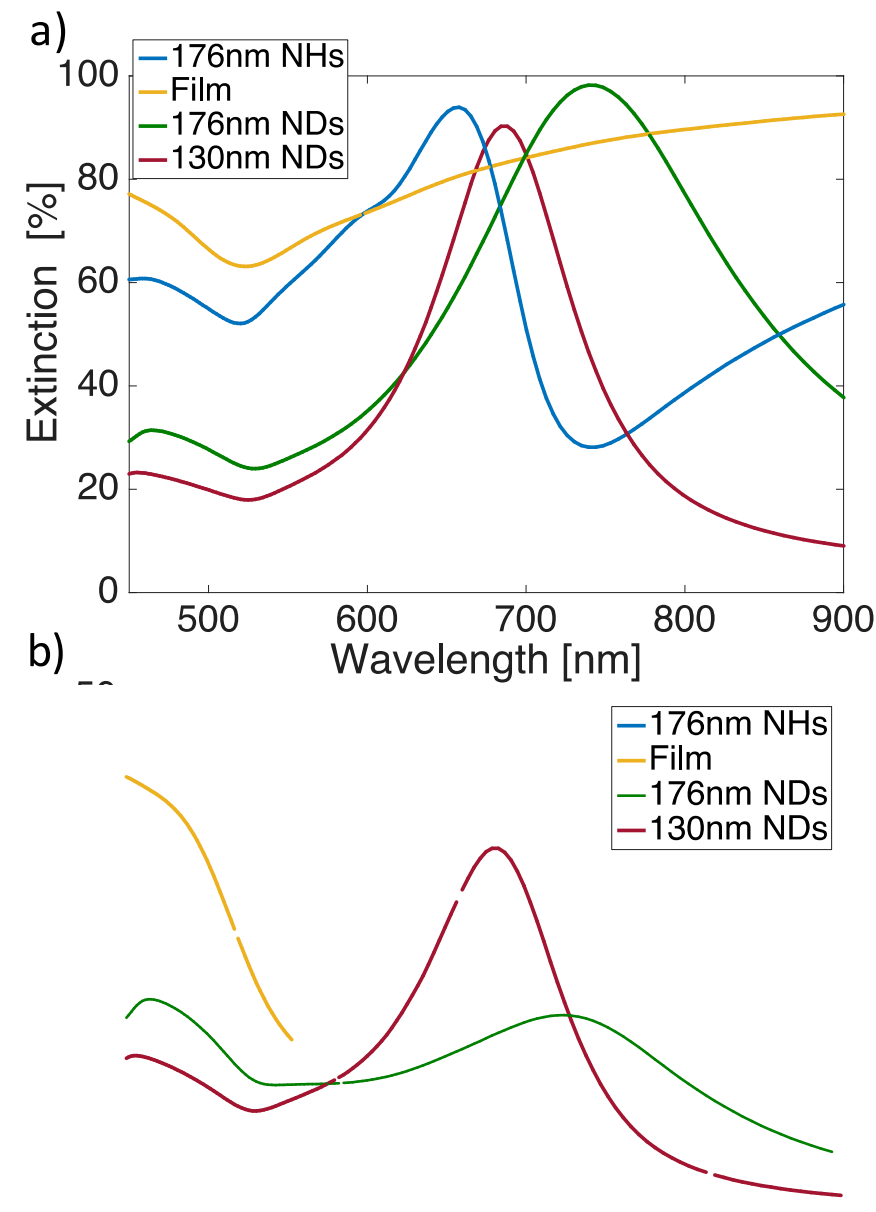

c)

Figure S1. (a) Extinction, (b) absorption and (c) reflection spectra of the four studied systems. 


\section{Integrated reflected power}

Following the same methodology as for the absorbed power (Figure 1d) and transmitted power (Figure 1e), we have calculated the total integrated reflected power (reflection + backscattering), in $\mathrm{W} / \mathrm{m}^{2}$ and in $\%$ upon solar illumination with an irradiance of 1 Sun $\left(1000 \mathrm{~W} / \mathrm{m}^{2}\right)$ (Figure S2).

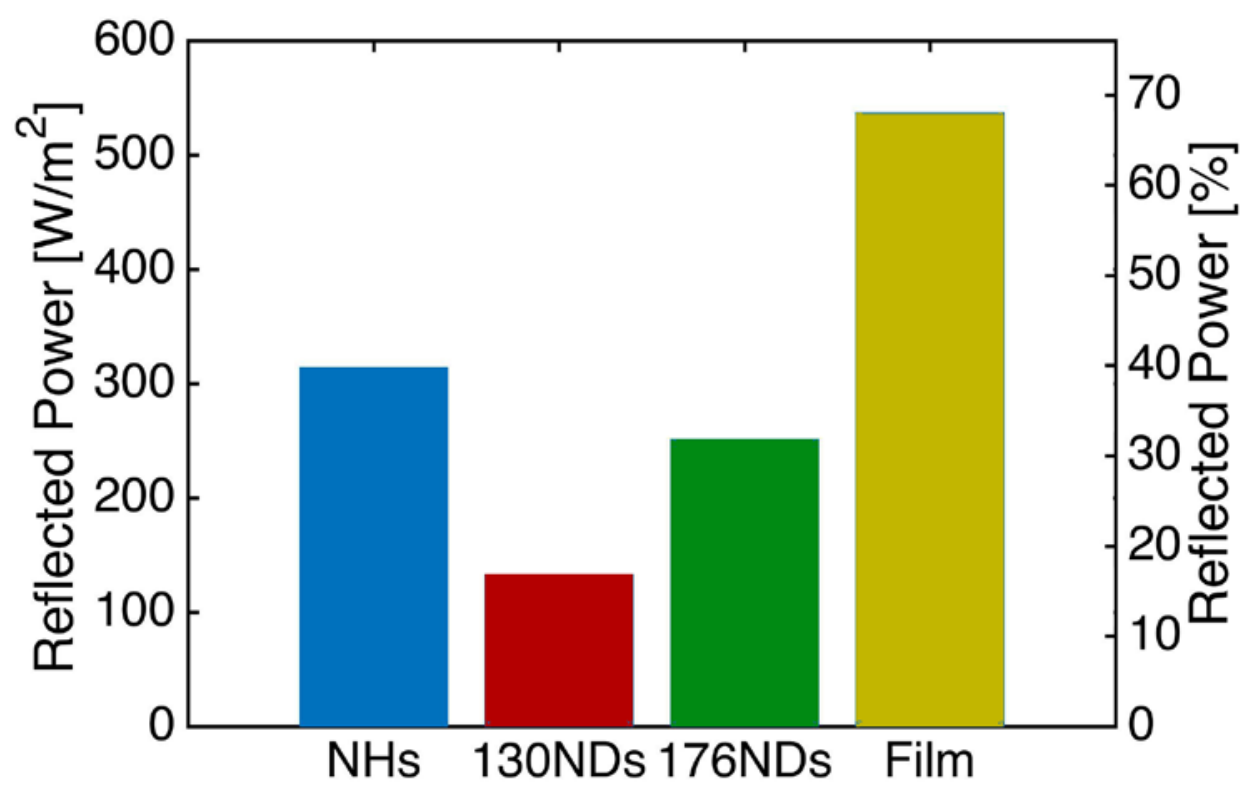

Figure S2. Reflected power for the four studied systems (305 nm spacing). 
Extinction of nanodisks and nanoholes and total absorbed power for nanoholes at different spacing

The extinction is simulated for $176 \mathrm{~nm}$ nanoholes and nanodisks at different spacings between 220 and $550 \mathrm{~nm}$ (Figure S3a and b). At higher spacing (1000 nm and above) the extinction for nanoholes start resembling those of a gold film (Figure S3c).

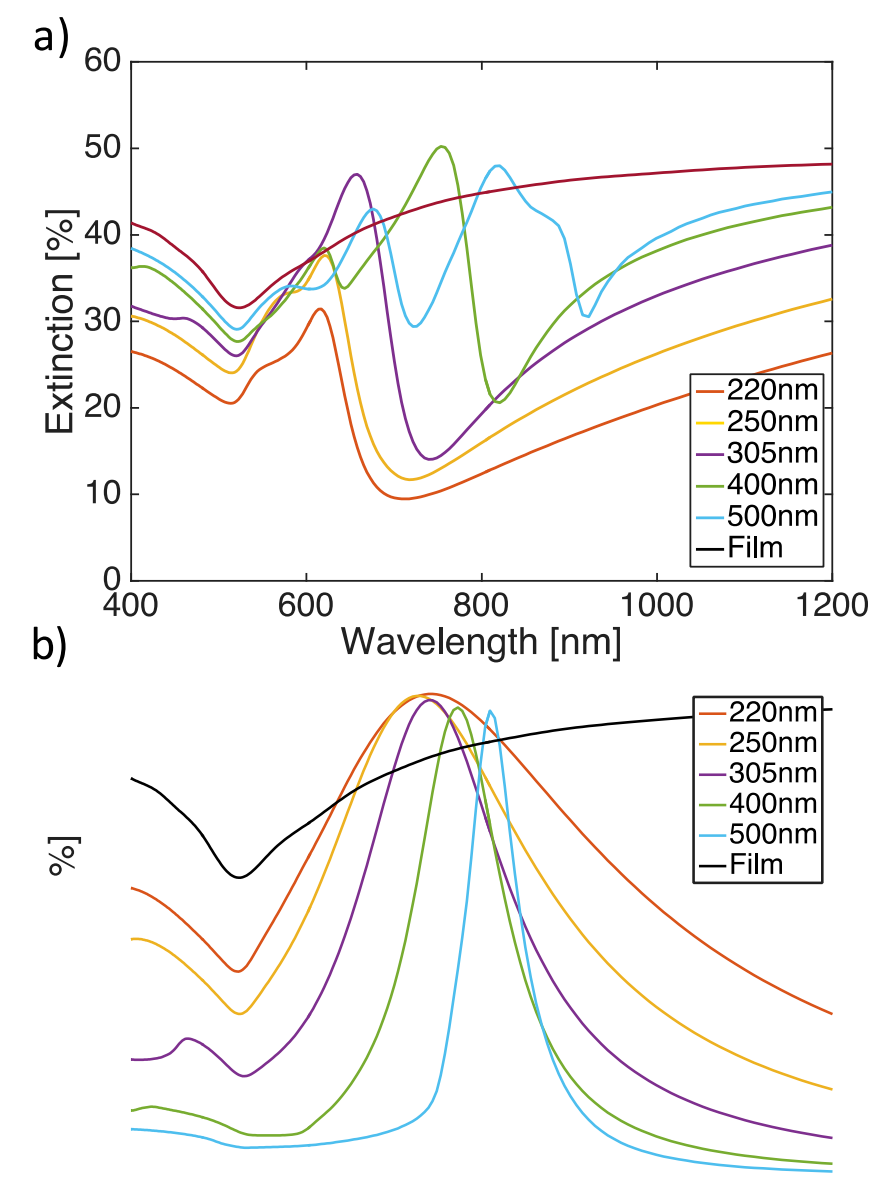


Figure S3. a) Extinction for the studied nanoholes at different characteristic spacing. b) Extinction for the $176 \mathrm{~nm}$ nanodisks at different characteristic spacing. c) Absorbed power for nanoholes at different spacings. 


\section{Radial distribution function}

We used SEM images to calculate the radial distribution function (Figure S4). The results show that there is a short-range order in the plasmonic nanohole arrays fabricated by colloidal lithography, with a characteristic spacing of $305 \mathrm{~nm}$.

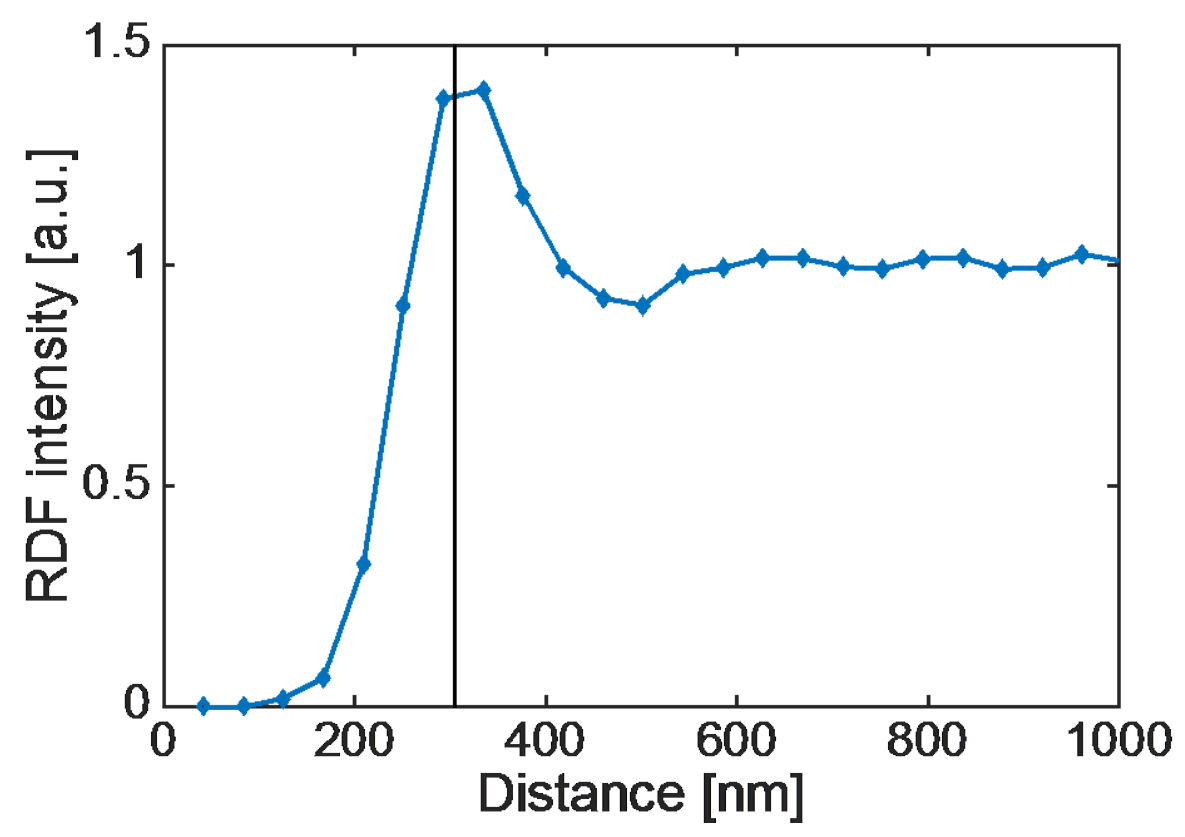

Figure S4. Radial distribution function for one of the fabricated plasmonic nanohole arrays. 


\section{Absorption directionality}

The optical absorption for gold nanohole arrays was simulated for front illumination and back illumination (from the glass).The results show that absorption is promoted for the back illumination (Figure S5).

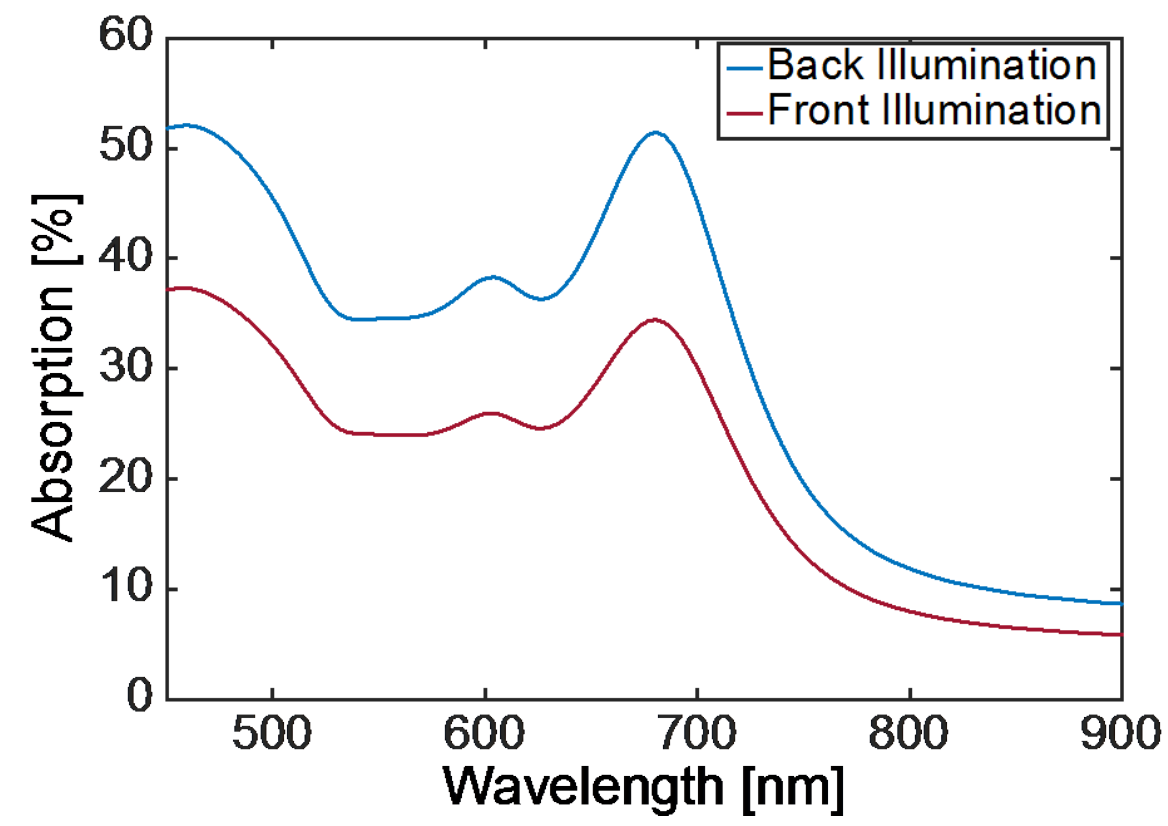

Figure S5. Simulated absorption using front (red) and back (blue) illumination for the nanohole array structure. 